\title{
1 Combined photoacoustic imaging to delineate the internal structure of paintings
}

\author{
3 Alice Dal Fovo, ${ }^{1,2, *, \dagger}$ [i] George J. Tserevelakis, ${ }^{3, \dagger}$ [i] Athanasia Papanikolaou, ${ }^{3}$ Giannis Zacharakis, ${ }^{3}$ \\ and Raffaella Fontana ${ }^{1}$ \\ ${ }^{1}$ Consiglio Nazionale delle Ricerche-Istituto Nazionale di Ottica (CNR-INO), Largo Enrico Fermi 6, 50125, Firenze, Italy \\ ${ }^{2}$ Dipartimento di Chimica, Università degli Studi di Firenze, Via della Lastruccia 3, 50019, Sesto Fiorentino, Firenze, Italy \\ ${ }^{3}$ Foundation for Research and Technology Hellas, Institute of Electronic Structure and Laser, N. Plastira 100, Heraklion, Crete, Greece \\ *Corresponding author: alice.dalfovo@ino.it
}

Received 28 November 2018; accepted 10 January 2019; posted 11 January 2019 (Doc. ID 352938); published 0 MONTH 0000

11 12
In this Letter, we present a combined photoacoustic imaging method, based on consecutive excitation using either the fundamental or the second-harmonic wavelength of a pulsed Nd:YAG laser for the stratigraphy of painted artworks. 2 Near-infrared excitation was employed for the imaging of hidden underdrawings in mock-up samples, whereas visible light for the thickness mapping of the overlying paint through the detection of photoacoustic signal attenuation. The proposed methodology was proven effective in measuring thick and strongly absorbing layers, which would not be possible by means of other pure optical techniques, while also enabling the visualization of features underneath the painted surface. Such an implementation expands significantly the applicability of the previously presented photoacoustic technique, which was limited to pointmeasurements, and paves the way for novel application in historical and technical studies, as well as in documenting restoring operations. (c) 2019 Optical Society of America

https://doi.org/10.1364/OL.99.099999

Laser-based optical techniques are becoming more and more significant in the field of art diagnostics, thanks to their capability to obtain chemical, structural, and morphological information in a non-invasive way. In regard to paintings, hidden features underneath the painted surface, i.e., underdrawings, signatures, pentimenti, ancient and modern materials, overpaintings, and varnishes can be measured without sampling or damaging artwork integrity. More specifically, restoring operations involving the selective removal of altered varnishes, such as the cleaning process, definitely benefit from the micrometric in-depth evaluation of the aged materials to be removed. In the last decades, several optical techniques have been applied for the non-invasive stratigraphic analysis of paintings [1-12]. Among others, optical coherence tomography (OCT) [1,2] provides high-resolution cross-sectional images for the visualization of low scattering varnish and semi-transparent paints, sometimes enabling the visualization of underdrawings [2]. More recently, nonlinear optical microscopy modalities [8-12] have been tested in painting diagnostics with the aim to extend maximum achievable imaging depth in highly scattering and semi-opaque materials.

In a previous study [13], we introduced a point-wise novel method called photoacoustic signal attenuation analysis (PAcSAA), which takes advantage of the superficial visible light absorption by the pigment particles for the estimation of paint layer local thickness. The technique is based on the photoacoustic effect, occurring when the absorption of intensity-modulated optical radiation by a medium induces the generation of broadband acoustic waves. Compared to visible and infrared radiation, the transmission of photoacoustic signals through scattering media is considerably higher [14], and characterized by a frequency-dependent exponential attenuation [15-18], which is significantly stronger for higher frequency components [15-17]. By analyzing the photoacoustic signal attenuation through a given material and by estimating the respective attenuation coefficients, it is possible to determine the thickness of an object $[18,19]$. In our last work, we employed PAcSAA for local thickness measurements on purposely prepared paint samples. To this aim, we shifted the detected photoacoustic signals from the time to frequency domain and used the amplitude spectrum for the estimation of the average transmitted frequency (ATF) as a characteristic measure of the overall acoustic attenuation during the signal propagation through the paint layer. ATF values were correlated with the thickness of different pigmented layers by fitting exponential decay models which, subsequently, were used to perform spot measurements on a painted canvas sample with high accuracy.

In this Letter, we present a combined photoacoustic imaging methodology employing consecutive excitation through the fundamental and second-harmonic wavelengths emitted by a pulsed Nd:YAG laser source, with the aim of delineating the stratigraphy of paintings. Therefore, we have introduced a novel imaging version of PAcSAA, called photoacoustic signal attenuation imaging (PACSAI), with optimized irradiation, acquisition, and scanning parameters, enabling the recording of accurate stratigraphic data in opaque paint layers. In principle, a stratigraphy of an object can be obtained by tuning the wavelength according to each layer and knowing the attenuation 
coefficients of the materials. In fact, by selecting proper excitation wavelengths, it is possible to confine the generation of photoacoustic waves to a particular layer, while enabling the transmission of the radiation through the other layers. In addition, the visualization of underdrawings can be also obtained by combining the area-wise PAcSAI modality with the near-infrared photoacoustic imaging [14], as shown in this Letter. Such in-depth and areal information may definitely turn useful to plan or monitor restoring operations, i.e., the cleaning process, and for historical and technical studies through the disclosure of painting internal features. The dual-content data have been compared with OCT images, validating the capabilities of the proposed diagnostic approach.

The photoacoustic imaging setup (Fig. 1) is based on a diode-pumped $Q$-switched Nd:YAG laser at $1064 \mathrm{~nm}$ (QIR-1064-200-S, CrystaLaser LC, Reno, Nevada; pulse energy, $29.4 \mu \mathrm{J}$; pulse duration, $\sim 8 \mathrm{~ns}$; selected repetition rate, $5 \mathrm{kHz} ; \mathrm{M}^{2}$ factor, 1.2) whose second harmonic (at $532 \mathrm{~nm}$ ) is generated by focusing the beam on a lithium triborate crystal. To switch between infrared and visible excitation, two bandpass filters (FL1064-10, Thorlabs, Newton, New Jersey and FF01-531/40-25, Semrock, Rochester, New York) are used, which are mounted on a filter wheel.

The beam was focused on the back side of the sample, at the interface between the paint layer and the substrate, by an air immersion objective lens (Achromat $8 \times$, LOMO, St. Petersburg, Russia; NA: 0.2), after proper attenuation (pulse 3 energy on the sample $\sim 200 \mathrm{~nJ}$ ). The sample was placed into an optically transparent Petri dish filled with a water-based gel of cellulose ether (carboxymethyl cellulose [CMC] gel-3\%) acting as a coupling medium to ensure an efficient transmission of the laser-induced ultrasound signal. CMC is well suited for artwork diagnostics: being an inert material, it is widely used for the cleaning of the painted surfaces due to its safety features, wide availability, and low cost. The Petri dish was fixed on a highprecision motorized XY micrometric stage (8MTF-75LS05, Standa, Vilinius, Lithuania) enabling the point-by-point scanning over the region of interest, whereas the focal plane

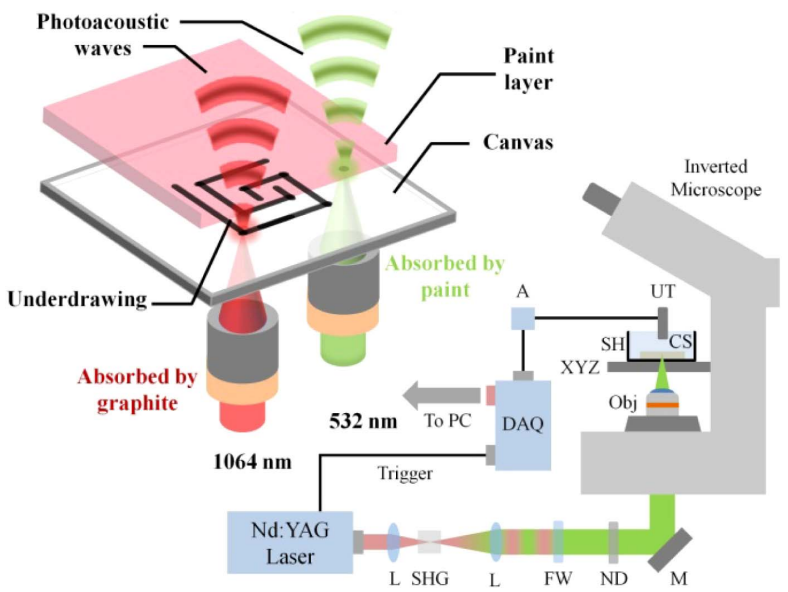

Fig. 1. Photoacoustic setup for the successive imaging of painting samples using two irradiation wavelengths. L, lens; SHG, secondharmonic generation crystal; FW, filter wheel; ND, neutral density filter; M, mirror; Obj, objective lens; XYZ, 3D translational stage; $\mathrm{SH}$, sample holder; CS, canvas sample; UT, ultrasonic transducer; A, amplifier; DAQ, data acquisition card. positioning was performed by the built-in manual Z-control of the microscope. For the detection of the generated photoacoustic waves, a single element spherically focused broadband ultrasonic transducer (HFM28, SONAXIS, Besancon, France; central frequency, $73 \mathrm{MHz}$; effective bandwidth, $\sim 90 \mathrm{MHz}$ at $-6 \mathrm{~dB}$; focal distance, $4.53 \mathrm{~mm}$ ) was immersed into CMC in a confocal and coaxial configuration with respect to the optical axis.

The combined photoacoustic imaging method was first tested on a multilayer sample consisting of a number of superimposed black tape layers producing an increasing thickness on a glass support (coverslip $2.5 \times 2.5 \mathrm{~cm} ; 150 \mu \mathrm{m}$ thick). This sample of well-defined structural, optical, and acoustic properties was purposely produced to test the performance of PAcSAI before proceeding to the analysis of more realistic cases, while clearly highlighting the capability of PAcSAI in probing highly turbid and thick materials, in contrast to OCT imaging. The thickness of each area (1-5 tape layers) was preliminarily assessed by a micro-profilometer (Perthometer S5P, Mahr, Göttingen, Germany) with one single tape measured at $110 \pm$ $5 \mu \mathrm{m}$ [Fig. 2(a)]. The sample was irradiated from the back side with the second harmonic of the Nd:YAG laser beam. The generated time-domain photoacoustic signal was recorded point by point over a scanning area of $16 \mathrm{~mm}^{2}$ resulting in a 200 by 200 pixels image.

Data were then processed in MATLAB and ImageJ environments for the reconstruction of the photoacoustic amplitude image [Fig. 2(a)] where, for each pixel, the brightness value corresponds to the maximum amplitude projection (MAP) obtained by averaging 128 waveforms, which are recorded for $\mathrm{S} / \mathrm{N}$ enhancement. The amplitude values were calculated as an average on selected areas of $\sim 4600$ pixels at the center of each region of different thickness, and then plotted as a function of thickness. The best fitting curve demonstrates an exponential decay of the photoacoustic amplitude with the propagation length through the sample's volume (decay

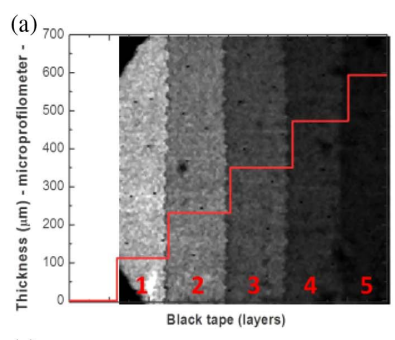

(c)
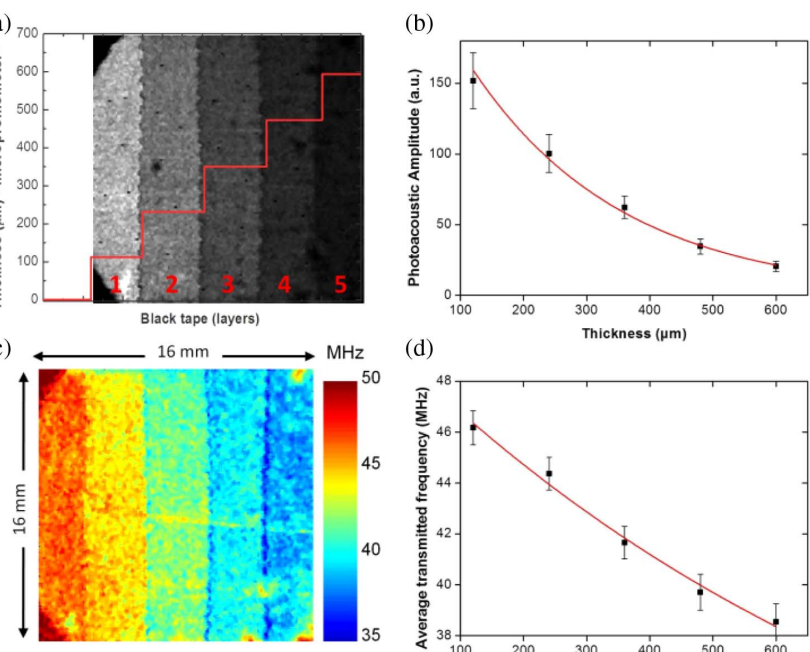

(d)

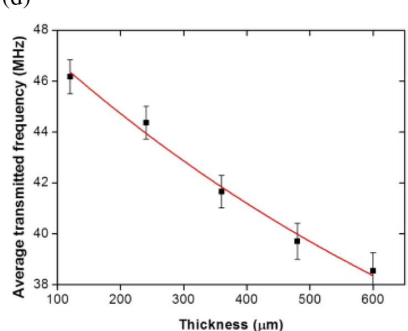

Fig. 2. PAcSAA of a black tape sample. (a) MAP photoacoustic image in grayscale showing the different layers, with the respective thickness values obtained with a microprofilometer (red line); (b) plot of the average photoacoustic amplitude as a function of thickness; (c) ATF image displayed within the range 35-50 MHz; and (d) plot of the average ATF values as a function of total layer thickness. 
constant: $4.18 \times 10^{-3}, R^{2}=0.992$ ) [Fig. 2(b)]. Finally, a respective ATF color-scale image was generated within the acoustic band of 10-100 MHz, following a fast Fourier transform of the acquired time-domain photoacoustic waveforms. Similar to the amplitude image, the ATF values were calculated as the average on the previously selected areas for each tape layer and plotted together with an exponential fitting curve (decay constant: $1.08 \times 10^{-3}, R^{2}=0.982$ ) as a function of layer thickness [Fig. 2(d)].

On the basis of these preliminary results, the effectiveness of PAcSAI was evaluated on an ad-hoc prepared sample simulating a real painting on canvas. A geometric pattern was drawn on the preparation layer covering the canvas support, using a graphite pencil. For the paint layer, we used primary red magenta PV19-73900 (Quinacridone $\left[\mathrm{C}_{2} \mathrm{OH}_{12} \mathrm{~N}_{2} \mathrm{O}_{2}\right]$, organic) extrafine acrylic color (Maimeri Brera, IT), as in our previous work [13].

The sample was also characterized by a spectral-domain OCT (Thorlabs Telesto-II, center wavelength, $1300 \mathrm{~nm}$; imaging depth, $3.5 \mathrm{~mm}$; axial resolution in air, $5.5 \mu \mathrm{m}$; lateral resolution, $7 \mu \mathrm{m}$ ); tomographic cubes $9 \mathrm{~mm} \times 9 \mathrm{~mm} \times 1 \mathrm{~mm}$ were acquired with sampling step of $5 \mu \mathrm{m}$ on $\mathrm{x}$ and $\mathrm{y}$, and $3.55 \mu \mathrm{m}$ on $\mathrm{z}$, enabling the visualization of the graphite underdrawing [Figs. 3(a) and 3(c)], the latter for comparison with the near-infrared photoacoustic imaging session. A clearer crosssectional visualization of the paint layer was obtained with a time-domain confocal-OCT prototype operating at $1550 \mathrm{~nm}$ (axial resolution, $10 \mu \mathrm{m}$ in air; lateral resolution, $2.5 \mu \mathrm{m}$ ), developed at the Istituto Nazionale di Ottica (CNR-INO) [2] scanning the surface along a line in its middle [light blue rectangle in Fig. 3(a)]. The image was acquired with 5 and $1 \mu \mathrm{m}$ sampling step in y and $\mathrm{z}$ directions (image size $25 \mathrm{~mm}$ in length
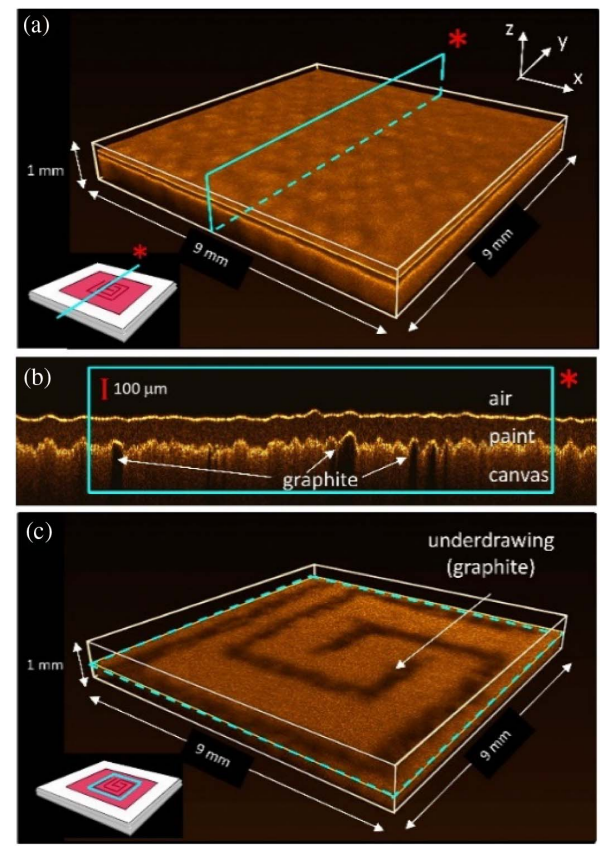
ness: $82 \pm 9 \mu \mathrm{m}$ ); and (c) $x-y$ section of the tomocube showing the underdrawing. and $1 \mathrm{~mm}$ in depth). The paint thickness was computed by averaging 12 measurements along the selected profile, resulting in $82 \pm 12 \mu \mathrm{m}$ [Fig. 3(b)].

Photoacoustic imaging with excitation at $1064 \mathrm{~nm}$ was applied for the visualization of the underdrawing. The sample was irradiated from its back side, and the raster scanning of a $10 \mathrm{~mm} \times 10 \mathrm{~mm}$ area (200 by 200 pixels, 750 averaging measurements per point) was performed. The photoacoustic waves are generated in correspondence of the underdrawing pattern, due to the high absorption of graphite in the near-infrared, and transmitted through the paint, which is transparent in this spectral region, thus revealing exclusively the hidden sketch [Fig. 4(a)].

Following the near-infrared photoacoustic imaging session, the evaluation of the paint's thickness was performed with PAcSAI [Fig. 4(b)]. The sample was scanned in the previously analyzed region using the $532 \mathrm{~nm}$ wavelength, by averaging over the same number of measurements for $\mathrm{S} / \mathrm{N}$ improvement. Since light at $532 \mathrm{~nm}$ is absorbed by both graphite and red paint, the photoacoustic waves are generated over the entire scanned area and are attenuated according to the local thickness of the paint layer. However, the attenuation is greater in correspondence of the underdrawing, due to the presence of two superimposed absorbing materials, making the underdrawing slightly visible in the final image. Differently, by irradiating at $1064 \mathrm{~nm}$, a much better image contrast is obtained due to the greater difference in the materials absorption. The two amplitude images acquired at 1064 and $532 \mathrm{~nm}$ were merged in one single image as blue and red channels, respectively [Fig. 4(c)]. Finally, to provide a map of paint thickness over the scanned region, we shifted the time-domain waveforms recorded at $532 \mathrm{~nm}$ to the frequency domain, and estimated the ATF values for each point within the range of 10-130 MHz. Acoustic signals originated by the underdrawing regions are excluded from the analysis, since they are generated through the absorption of light by the graphite, rather than the

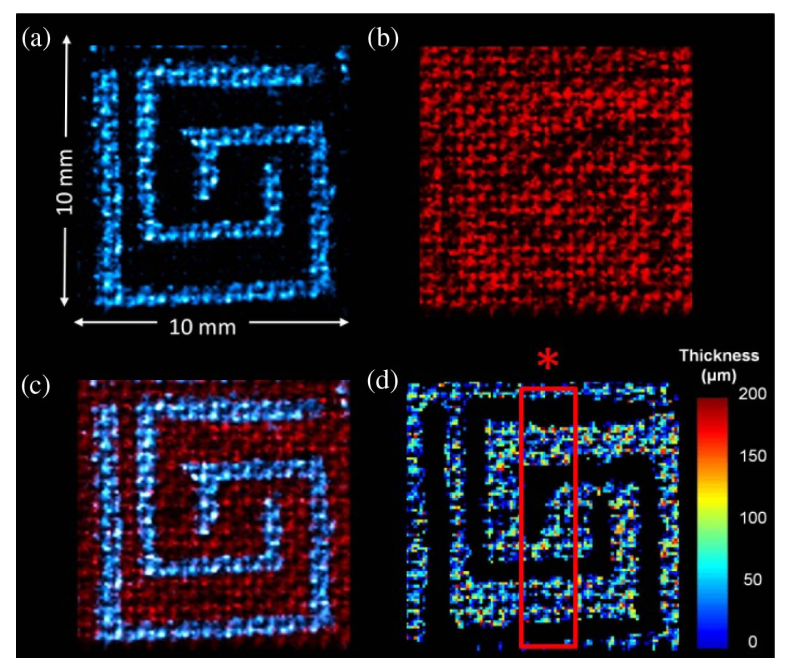

Fig. 4. Combined photoacoustic imaging results: (a) MAP photoacoustic image at $1064 \mathrm{~nm}$ (blue channel); (b) MAP image at $532 \mathrm{~nm}$ (red channel); (c) combined image (MAP at 1064 and $532 \mathrm{~nm}$ ); and (d) PAcSAI image with estimated paint thickness values on the color scale. The considered area for mean thickness measurement is highlighted by the red rectangle*. 
overlying paint. We used the exponential decay fitting parameters previously defined for primary red magenta [13] to relate the estimated ATF values (megahertz) with layer thickness $\mathrm{x}(\mu \mathrm{m})$ according to the equation

$$
x=-529 \times \ln \left(\frac{\mathrm{ATF}+0.03}{59.82}\right) \text {, }
$$

generating a PAcSAI map corresponding to the overlying paint layer thickness [Fig. 4(d)].

To validate the capabilities of PAcSAI for thickness measurements in paint layers, we have estimated the mean thickness on a representative area [outlined by the red rectangle in Fig. 4(d)] providing results with adequate statistical significance, and compared it with the recorded OCT profiles. It has to be noted that all extracted values smaller than the theoretical axial resolution of the transducer $(\sim 15 \mu \mathrm{m})$ [20] were excluded from the analysis as measurement artifacts. The obtained thickness for the selected area (686 pixels) was $77.8 \pm 1.9 \mu \mathrm{m}$, where measurement uncertainty corresponds to the standard error of the mean. PAcSAI results are in good agreement with OCT thickness $(\sim 82 \mu \mathrm{m})$, demonstrating the reliability of the proposed technique.

The combined photoacoustic imaging methodology has proven to represent a promising diagnostic tool for the analysis of painted artworks. The possibility of high-resolution imaging to disclose the underdrawing, as well as the stratigraphic information of paint layers, using the same experimental setup, turns definitely useful in the case of conservation studies and restoring operations. The fundamental and second-harmonic wavelengths of a single laser source enable the application of the presented method to a variety of drawing materials and paint layers containing different pigments and binding media. Moreover, displaying the results as images eases their interpretation by final users (restorers, conservators, art historians) directly involved in conservation interventions. In view of future applications on more complex cases, i.e., real objects with multiple paint layers of different pigments or paint mixtures, both the experimental apparatus and data processing have to be implemented. For example, a multispectral photoacoustic imaging approach could be used to excite efficiently a specific paint layer by tuning an appropriate irradiation wavelength. For paint mixtures, the exponential decay parameters could be estimated as the weighted average of the acoustic attenuation coefficients of individual paints, according to their light absorption properties. Furthermore, the system's performance could be enhanced in terms of spatial and temporal resolution (e.g., high frequency transducers, fast repetition rate lasers, galvo scanners), by taking into account the total time of flight of the recorded photoacoustic signal for each point. Finally, a pure acoustic imaging technique (e.g., a pulse-echo modality),

using the same ultrasonic transducer, could provide additional contrast to the combined photoacoustic imaging modality. These upgrades well represent the potential of the proposed technique and constitute objects of future research work.

276

277

278

279

Funding. Horizon 2020 Framework Programme (H2020) (654028, 654148); FP7 People: Marie-Curie Actions (PEOPLE) (PITN-GA-2012-317526); Stavros Niarchos Foundation (SNF) (MIS 5002478, MIS5002755).

${ }^{\dagger}$ These authors contributed equally to this Letter.

280 281 282 283

\section{REFERENCES}

1. P. Targowski, M. Gòra, and M. Wojtkowski, Laser Chem. 2006, 35373 (2006).

2. R. Fontana, A. Dal Fovo, J. Striova, L. Pezzati, E. Pampaloni, M. Raffaelli, and M. Barucci, Appl. Phys. A 121, 957 (2015).

3. B. Blümich, J. Perlo, and F. Casanova, Prog. Nucl. Magn. Reson. Spectrosc. 52, 197 (2008).

4. N. Proietti, D. Capitani, and V. Di Tullio, Sensors 14, 6977 (2014).

5. A. J. L. Adam, P. C. M. Planken, S. Meloni, and J. Dik, Opt. Express 17, 3407 (2009).

6. G. Filippidis, M. Massaouti, A. Selimis, E. J. Gualda, J. M. Manceau, and S. Tzortzakis, Appl. Phys. A 106, 257 (2012).

7. H. Liang, M. Mari, C. S. Cheung, S. Kogou, P. Johnson, and G. Filippidis, Opt. Express 25, 19640 (2017).

8. G. Latour, J. P. Echard, M. Didier, and M. C. Schanne-Klein, Opt. Express 20, 24623 (2012).

9. G. Filippidis, G. T. Tserevelakis, A. Selimis, and C. Fotakis, Appl. Phys. A 118, 417 (2015).

10. M. Oujja, S. Psilodimitrakopoulos, E. Carrasco, M. Sanz, A. Philippidis, A. Selimis, P. Pouli, G. Filippidis, and M. Castillejo, Phys. Chem. Chem. Phys. 19, 22836 (2017).

11. T. E. Villafana, W. P. Brown, J. K. Delaney, M. Palmer, W. S. Warren, and M. C. Fischer, Proc. Natl. Acad. Sci. USA 111, 1708 (2014).

12. A. Dal Fovo, R. Fontana, J. Striova, E. Pampaloni, M. Barucci, M. Raffaelli, R. Mercatelli, L. Pezzati, and R. Cicchi, in Proceedings of the International Conference LACONA XI (2017).

13. G. J. Tserevelakis, A. Dal Fovo, K. Melessanaki, R. Fontana, and G. Zacharakis, J. Appl. Phys. 123, 123102 (2018).

14. G. J. Tserevelakis, I. Vrouvaki, P. Siozos, K. Melessanaki, K Hatzigiannakis, C. Fotakis, and G. Zacharakis, Sci. Rep. 7, 747 (2017).

15. B. E. Treeby and B. T. Cox, Proc. SPIE 7177, 717716 (2009).

16. S. R. Cherry, R. D. Badawi, and J. Qi, Essentials of In Vivo Biomedical Imaging (CRC Press, 2016), p. 105.

17. X. L. Deán-Ben, D. Razansky, and V. Ntziachristos, Phys. Med. Biol. 56, 6129 (2011).

18. H. Ammari, E. Bretin, V. Jugnon, and A. Wahab, Mathematical Modeling in Biomedical Imaging II (Springer, 2012).

19. H. Ammari, E. Bretin, J. Garnier, and A. Wahab, Mathematical and Statistical Methods for Imaging (American Mathematical Society, 2011).

20. J. Yao and L. V. Wang, Laser Photonics Rev. 7, 758 (2013).

\section{5}

286

287

288

289

290

291

292

293

294

295

296

297

298

299

300

301

302

303

304

305

306

307

308

309

310

311

312

313

314

315

316

317

318

319

320

321

322

323

324

325

326 


\section{Queries}

1. AU: A mismatch has been discovered between the e-mail address in the manuscript and the e-mail address in OSA's system. Please let us know if the e-mail address in the manuscript should be changed.

2. AU: In the sentence that begins "Near infrared excitation was," it seems as there is a word(s) missing in the phrase "whereas visible light for the thickness mapping of the overlying paint through the detection of photoacoustic signal attenuation". Please check.

3. AU: Please verify the edits made in the sentence that begins "The sample was placed".

4. AU: The funding information for this article has been generated using the information you provided to OSA at the time of article submission. Please check it carefully. If any information needs to be corrected or added, please provide the full name of the funding organization/institution as provided in the CrossRef Open Funder Registry (https://search.crossref.org/funding).

\section{ORCID Identifiers}

The following ORCID identifiers were supplied for the authors of this article. Please review carefully. If changes are required, or if you are adding IDs for authors that do not have them in this proof, please submit them with your corrections for the article. Authors who do not have iDs on the proof may add them by logging into their OSA account. To do this, click on the "Update Account" link on your Prism homepage or log-in directly to http://account.osa.org, then click the button "Create or Connect your ORCID iD" in the ORCID section of the Participation tab. If the ORCID window does not appear, then change your browser settings to enable pop-ups. Please indicate in your corrections if you or any coauthors have done this. Each individual author is responsible for adding or correcting his or her $\mathrm{iD}$ using the steps outlined above.

- Alice Dal Fovo https://orcid.org/0000-0001-7960-2546

- George J. Tserevelakis https://orcid.org/0000-0002-9938-3472 